\title{
The Height Amplitudes of Surface Roughness based on Area-direction Character and Relative Assessed Indexes
}

\author{
Hongcai Wang ${ }^{1,2, a}$ and Yang Wang ${ }^{1, b}$ \\ ${ }^{1}$ School of Mechatronics Engineering, Harbin Institute of Technology, Harbin, Heilongjiang, 150001, \\ China \\ ${ }^{2}$ Key Laboratory of Mechanics in Advanced Manufacturing, Institute of Mechanics, Chinese \\ Academy of Sciences, Beijing, 100190, China \\ ahcwang@imech.ac.cn, bwyh@@hit.edu.cn
}

Keywords: Surface Roughness, Area-direction Character, Evaluation.

\begin{abstract}
To study the relationship among the height amplitude of surface roughness , assessed area and assessed direction, the height amplitudes $S R a, S R q, S R s k$ and $S R k u$ based on area-direction character are defined in the paper. And on the foundation of these parameters, relative assessed area indexes, assessed direction indexes and assessed total indexes are established. $S R a, S R q$, $S R s k$ and $S R k u$ are the natural extension of 2D height amplitudes $R a, R q, R s k$ and $R k u$. They are different from 3D height amplitudes $S a, S q$, Ssk and $S k u$. They provide an evidence to study the quantitative relationship among surface roughness, assessed area and assessed direction. Relative assessed area indexes, assessed direction indexes and assessed total indexes can be used to assess the distribution situation of height amplitudes of surface roughness related to area, direction and entireness.
\end{abstract}

\section{Introduction}

Presently, there are mainly 5 types of assessing methods of the surface roughness. They are 2D parameter assessing method, 3D parameter assessing method, fractal assessing method, graphics assessing method and image assessing method. In image assessing method, digital image such as color image, gray image or black-and-white image(two value image) are used to describe 3D curved surface[1,2].

In essence, the normal engineering has a 3D tiny structure. So $2 \mathrm{D}$ profile cannot comprehensively and accurately describe the surface of the parts.

Since seventies of twentieth century, a lot of researches have been made, and lot of assessing methods and assessing parameters of the surface roughness have been brought up[3,4]. But up to the present, uniform international standard has not formed yet.

Actual engineering surface profile has a close relationship with assessed area and assessed direction. Experimental result show that the difference of parameters from different profile on the same surface can attain to $50 \%$. In existing 3D parameter assessing methods, the different direction and different area are not considered when determine datum plane. So the relationship among the surface roughness, assessed area and assessed direction cannot be described.

The height amplitudes are the basic kind of parameters in surface roughness. Existing 3D height amplitudes conclude surface arithmetic mean deviation $S a$, surface root mean square deviation $S q$, surface skewness $S s k$, surface kurtosis $S k u$, etc. To study the relationship among the height amplitude of surface roughness, assessed area and assessed direction, four types of height amplitudes based on area-direction character including $S R a, S R q, S R s k$ and $S R k u$ are defined in the paper. And on the foundation of these parameters, relative assessed indexes are established. 


\section{Height amplitudes based on area-direction character}

General 3D assessed parameters such as $S a, S q$, Ssk and $S k u$ cannot reflect and assess directional character, because there is only one benchmark, that is, datum plane. Statistical method in this paper is to average directional profile family parameters twice. The procedure of the method is as following.

First, calculate each datum line of $\mathrm{n}$ profiles in same direction in assessed area, and calculate $R a$, $R q, R s k$ and $R k u$.

Second, calculate directional assessed parameter $S R a, S R q, S R s k$ and $S R k u$ in this assessed area by averaging $R a, R q$, $R s k$ and $R k u$.

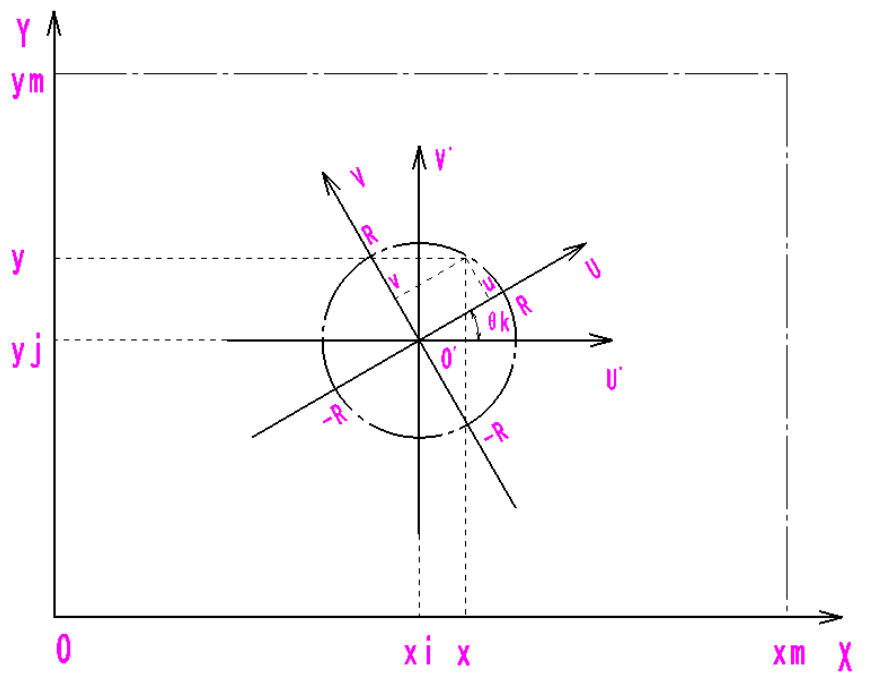

Fig. 1 Assessed coordinate system

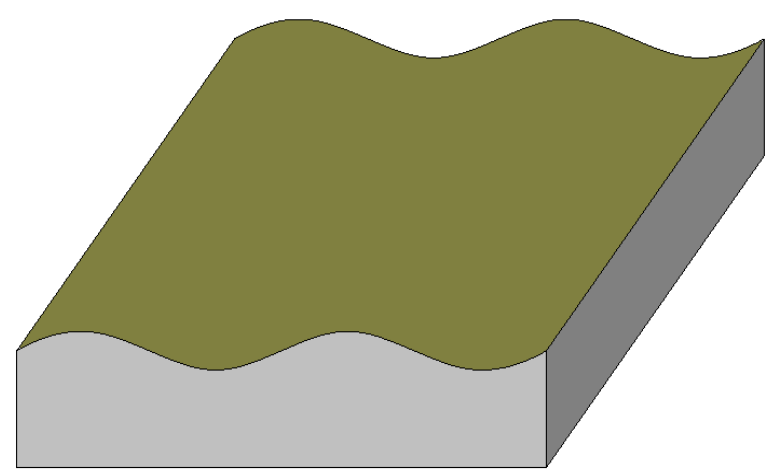

Fig. 2 Ideal cylindrical diagram

Figure 1 is assessed coordinate system. OXYZ is base coordinate system( $Z$ axis is vertical paper outward). Rectangle $\mathrm{xm} \times \mathrm{ym}$ is assessed surface. Height amplitude coordinate $z(x, y)$ on the surface is known. $\left(x_{i}, y_{j}\right)$ is assessed area center coordinate. $\theta_{k}$ is assessed angle. O' $\mathrm{U}^{\prime} \mathrm{V}^{\prime} \mathrm{W}$ ' is assessed position coordinates(W' axis is vertical paper outward). O'UVW is assessed direction coordinates(W axis is vertical paper outward) on which $w(u, v)$ is height amplitude coordinate. The assessed area is a circle which center is O', and radius is $R .(u, v)$ and $(x, y)$ is the same point. There is a relationship as following.

$$
\left[\begin{array}{l}
x \\
y
\end{array}\right]=\left[\begin{array}{cc}
\cos \theta_{k} & -\sin \theta_{k} \\
\sin \theta_{k} & \cos \theta_{k}
\end{array}\right]\left[\begin{array}{l}
u \\
v
\end{array}\right]+\left[\begin{array}{l}
x_{i} \\
y_{j}
\end{array}\right], w(u, v)=z(x, y) .
$$

Suppose $T(u, v)=w(u, v)-\frac{1}{f 2(v)-f 1(v)} \int_{f 1(v)}^{f 2(v)} w(u, v) d u$.

The definitions of the height amplitudes based on area-direction character are:

$$
\begin{aligned}
& \operatorname{SRa}(l)=\operatorname{SR} a\left(x_{i,} y_{j}, \theta_{k}\right)=\frac{1}{2 R} \int_{-R}^{R}\left[\frac{1}{f 2(v)-f 1(v)} \int_{f 1(v)}^{f 2(v)}|T(u, v)| d u\right] d v . \\
& S R q(l)=S R q\left(x_{i}, y_{j}, \theta_{k}\right)=\frac{1}{2 R} \int_{-R}^{R}\left[\sqrt{\frac{1}{f 2(v)-f 1(v)} \int_{f 1(v)}^{f 2(v)} T(u, v)^{2} d u}\right] d v . \\
& \operatorname{SRsk}(l)=\operatorname{SRsk}\left(x_{i}, y_{j}, \theta_{k}\right)=\frac{1}{2 R} \int_{-R}^{R}\left\{\frac{\frac{1}{f 2(v)-f 1(v)} \int_{f 1(v)}^{f 2(v)} T(u, v)^{3} d u}{\left[\sqrt{\frac{1}{f 2(v)-f 1(v)} \int_{f 1(v)}^{f 2(v)} T(u, v)^{2} d u}\right]^{3}}\right\} d v . \\
& \operatorname{SRku}(l)=\operatorname{SRku}\left(x_{i}, y_{j}, \theta_{k}\right)=\frac{1}{2 R} \int_{-R}^{R}\left\{\frac{\frac{1}{f 2(v)-f 1(v)} \int_{f 1(v)}^{f 2(v)} T(u, v)^{4} d u}{\left[\frac{1}{f 2(v)-f 1(v)} \int_{f 1(v)}^{f 2(v)} T(u, v)^{2} d u\right]^{2}}\right\} d v .
\end{aligned}
$$


In these formula, $i=1,2 \ldots r$ is the number of horizontal areas.

$j=1,2 \ldots s$ is the number of vertical areas.

$k=1,2 \ldots t$ is the number of directions.

$l=i+r \times j+r \times s \times k$ is the number of areal directions.

$l=k+t \times i+t \times r \times j$ is the number of directional areas.

$f 1(v)=-\sqrt{R^{2}-v^{2}}, f 2(v)=\sqrt{R^{2}-v^{2}}$.

$S R a(l)$ is area-directional surface arithmetic mean deviation.

$S R q(l)$ is area-directional surface root mean square deviation.

$\operatorname{SRsk}(l)$ is area-directional surface skewness.

$S R k u(l)$ is area-directional surface kurtosis.

The rationality of these parameters can be preliminary validated by using ideal Cylinder. Figure 2 is an ideal Cylindrical diagram. In the direction of generatrix,

$S R a=R a_{1}=\ldots=R a_{n}=0, S R q=R q_{1}=\ldots=R q_{n}=0$.

That is, original $2 \mathrm{D}$ assessed parameters are equal to $3 \mathrm{D}$ assessed parameters upwards. They are all equal to zero.

In the directional of directrix in cylinder,

$S R a=R a_{1}=\ldots=R a_{n}=S a, S R q=R q_{1}=\ldots=R q_{n}=S q$.

That is, original $2 \mathrm{D}$ assessed parameters and original $3 \mathrm{D}$ assessed parameters are equal to $3 \mathrm{D}$ assessed parameters upwards.

$S R a, S R q, S R s k$ and $S R k u$ can be regarded as relative extension of $R a, R q, R s k$ and $R k u$ to 3D assessed parameters.

\section{Assessed indexes}

Assessed area indexes. Assessed area indexes are used to describe the distribution situation of height amplitudes in each assessed area in special assessed direction. The definitions are:

$$
\begin{aligned}
& S R a_{a \min }(m)=\min \left[\operatorname{SRa}\left(x_{i,}, y_{j}, \theta_{k}\right)\right] . \\
& S R a_{a \max }(m)=\max \left[\operatorname{SRa}\left(x_{i}, y_{j}, \theta_{k}\right)\right] . \\
& S R a_{\text {arange }}(m)=\operatorname{SRa} a_{a \max }(m)-S R a_{a \text { min }}(m) . \\
& S R a_{\text {amean }}(m)=\frac{1}{r \times s} \sum_{i=1}^{r} \sum_{j=1}^{s} \operatorname{SRa}\left(x_{i,} y_{j}, \theta_{k}\right) . \\
& S R a_{\text {astd }}(m)=\sqrt{\frac{1}{r \times s} \sum_{i=1}^{r} \sum_{j=1}^{s} \operatorname{SRa}\left(x_{i}, y_{j}, \theta_{k}\right)^{2}-S R a_{\text {amean }}(m)^{2}} . \\
& S R a_{\text {acv }}(m)=\frac{S R a_{\text {astd }}(m)}{S R a_{\text {amean }}(m)} .
\end{aligned}
$$

In these formula, $i=1,2 \ldots r$ is the number of horizontal areas.

$j=1,2 \ldots s$ is the number of vertical areas.

$k=1,2 \ldots t$ is the number of directions.

$S R a_{a \min }(m)$ is the minimum areal surface arithmetic mean deviation in assessed direction.

$S R a_{\text {amax }}(m)$ is the maximum areal surface arithmetic mean deviation in assessed direction.

$S R a_{\text {arange }}(m)$ is the range of areal surface arithmetic mean deviation in assessed direction.

$S R a_{\text {amean }}(m)$ is the mean deviation of areal surface arithmetic mean deviation in assessed direction.

$S R a_{a s t d}(m)$ is the STD of areal surface arithmetic mean deviation in assessed direction.

$S R a_{a c v}(m)$ is the $\mathrm{CV}$ of areal surface arithmetic mean deviation in assessed direction.

Assessed indexes of surface root mean square deviation, surface skewness, surface kurtosis in assessed direction can be calculated by replacing $S R a$ in the formula above-mentioned with $S R q, S R s k$ and $S R k u$. Standard deviation and extreme deviation are absolute values. Coefficient of variation is a relative value. 
Assessed direction indexes. Assessed direction indexes are used to describe the distribution situation of height amplitudes in each assessed direction in special assessed area. The definitions are:

$$
\begin{aligned}
& S R a_{d \min }(n)=\min \left[\operatorname{SRa}\left(x_{i,} y_{j}, \theta_{k}\right)\right] \text {. } \\
& S R a_{d \max }(n)=\max \left[\operatorname{SRa}\left(x_{i,} y_{j}, \theta_{k}\right)\right] \text {. } \\
& S R a_{\text {drange }}(n)=S R a_{d \max }(n)-S R a_{d \min }(n) \text {. } \\
& S R a_{\text {dmean }}(n)=\frac{1}{t} \sum_{k=1}^{t} \operatorname{SRa}\left(x_{i,} y_{j}, \theta_{k}\right) \text {. } \\
& S R a_{d s t d}(n)=\sqrt{\frac{1}{t} \sum_{k=1}^{t} S R a\left(x_{i}, y_{j}, \theta_{k}\right)^{2}-S R a_{d m e a n}(n)^{2}} \text {. } \\
& S R a_{d c v}(n)=\frac{S R a_{d s t d}(n)}{S R a_{d m e a n}(n)} \text {. }
\end{aligned}
$$

In these formula, $i=1,2 \ldots r$ is the number of horizontal areas.

$j=1,2 \ldots s$ is the number of vertical areas.

$k=1,2 \ldots t$ is the number of directions.

$n=i+r \times j$ is the number of areas.

$S R a_{\operatorname{dmin}}(n)$ is the minimum directional surface arithmetic mean deviation in assessed area.

$S R a_{\text {dmax }}(n)$ is the maximum directional surface arithmetic mean deviation in assessed area.

$\operatorname{Sra}_{\text {drange }}(n)$ is the range of directional surface arithmetic mean deviation in assessed area.

$S R a_{\text {dmean }}(n)$ is the mean deviation of directional surface arithmetic mean deviation in assessed area.

$S R a_{d s t d}(n$ is the STD of directional surface arithmetic mean deviation in assessed area.

$S R a_{d c v}(n)$ is the CV of directional surface arithmetic mean deviation in assessed area.

Assessed indexes of surface root mean square deviation, surface skewness, surface kurtosis in assessed area can be calculated by replacing $S R a$ in the formula above-mentioned with $S R q$, $S R s k$ and SRku.

Assessed total indexes. Assessed total indexes are used to describe the whole distribution situation of height amplitudes. The definitions are:

$$
\begin{aligned}
& S R a_{\text {min }}=\min \left[\operatorname{SRa}\left(x_{i,} y_{j}, \theta_{k}\right)\right] . \\
& S R a_{\text {max }}=\max \left[\operatorname{SRa}\left(x_{i,} y_{j}, \theta_{k}\right)\right] . \\
& S R a_{\text {range }}=S R a_{\text {max }}-S R a_{\min } . \\
& S R a_{\text {mean }}=\frac{1}{r \times S \times t} \sum_{i=1}^{r} \sum_{j=1}^{s} \sum_{k=1}^{t} S R a\left(x_{i,} y_{j}, \theta_{k}\right) . \\
& S R a_{\text {std }}=\sqrt{\frac{1}{r \times S \times t} \sum_{i=1}^{r} \sum_{j=1}^{s} \sum_{k=1}^{t} S R a\left(x_{i}, y_{j}, \theta_{k}\right)^{2}-S R a_{\text {mean }}{ }^{2} .} \\
& S R a_{c v}=\frac{S R a_{s t d}}{S R a_{\text {mean }}} . \\
& S R a_{a c v m}=\frac{1}{t} \sum_{m=1}^{t} S R a_{a c v}(m) . \\
& S R a_{d c v m}=\frac{1}{r \times s} \sum_{n=1}^{r \times s} S R a_{d c v}(n) .
\end{aligned}
$$

In these formula, $i=1,2 \ldots r$ is the number of horizontal areas.

$j=1,2 \ldots s$ is the number of vertical areas.

$k=1,2 \ldots t$ is the number of directions.

$n=i+r \times j$ is the number of areas.

$S R a_{\text {min }}$ is the minimum total surface arithmetic mean deviation.

$S R a_{\max }$ is the maximum total surface arithmetic mean deviation.

$S R a_{\text {range }}$ is the range of total surface arithmetic mean deviation. 
$S R a_{\text {mean }}$ is the mean deviation of total surface arithmetic mean deviation.

$S R a_{\text {std }}$ is the STD of total surface arithmetic mean deviation.

$S R a_{c v}$ is the CV of total surface arithmetic mean deviation.

$S R a_{a c v m}$ is the mean CV of total surface arithmetic mean deviation in area.

$S R a_{d c v m}$ is the mean CV of total surface arithmetic mean deviation in direction.

Assessed indexes of surface root mean square deviation, surface skewness, surface kurtosis in total can be calculated by replacing $S R a$ in the formula above-mentioned with $S R q, S R s k$ and $S R k u$.

\section{Conclusions}

$S R a, S R q, S R s k$ and $S R k u$ are the natural extension of 2D height amplitudes $R a, R q, R s k$ and $R k u$. They are different from 3D height amplitudes $S a, S q$, Ssk and $S k u$. They provide an evidence to study the quantitative relationship among surface roughness, assessed area and assessed direction. Relative assessed area indexes, assessed direction indexes and assessed total indexes can be used to assess the distribution situation of height amplitudes of surface roughness related to area, direction and entireness.

$S R a, S R q, S R s k$ and $S R k u$ have a close relationship with assessed area(include square and center position) and assessed direction. The assessed indexes have close relationship with the number of assessed area and the number of assessed direction. So the assessment should be used under the same condition.

\section{References}

[1] Kayahan Ersin, Oktem Hasan, Hacizade Fikret, et al. Measurement of Surface Roughness of Metals Using Binary Speckle Image Analysis[J]. Tribology International, 2010, 43(1-2): 307311.

[2] Zhongxiang Hu, Lei Zhu, Jiaxu Teng, et al. Evaluation of Three-dimensional Surface Roughness Parameters Based on Digital Image Processing[J]. International Journal of Advanced Manufacturing Technology, 2009, 40(3-4): 342-348.

[3] Zhihang Zhang. Micro-WEDM Three-dimensional Surface Morphology and Functions of the Research Assessment[D]. Harbin: Harbin Engineering University, 2009(In Chinese).

[4] W. P. Dong, E. Mainsah, K. J. Stout. Reference Planes for the Assessment of Surface Roughness in Three Dimensions[J]. Int. J. Mach. Tools Manufact, 1994, 35(2): 263-271. 
Advanced Composite Materials

10.4028/www.scientific.net/AMR.482-484

The Height Amplitudes of Surface Roughness Based on Area-Direction Character and Relative Assessed Indexes

10.4028/www.scientific.net/AMR.482-484.1150 